DESY 16-018, HU-EP-16/02, TTP16-004

\title{
Electron contribution to the muon anomalous magnetic moment at four loops
}

\author{
Alexander Kurz ${ }^{a, b}$, Tao Liu ${ }^{c}$, Peter Marquard ${ }^{b}$, Alexander V. Smirnov ${ }^{d}$, \\ Vladimir A. Smirnov ${ }^{e, f}$, Matthias Steinhauser ${ }^{a}$
}

(a) Institut für Theoretische Teilchenphysik, Karlsruhe Institute of Technology (KIT), 76128 Karlsruhe, Germany

(b) Deutsches Elektronen Synchrotron DESY, Platanenallee 6

15738 Zeuthen, Germany

(c) Department of Physics, University of Alberta, Edmonton AB T6G 2J1, Canada

(d) Scientific Research Computing Center, Moscow State University, 119991, Moscow, Russia

(e) Skobeltsyn Institute of Nuclear Physics of Moscow State University, 119991, Moscow, Russia

(f) Institut für Mathematik und Institut für Physik, Humboldt-Universität zu Berlin, 12489 Berlin, Germany

\begin{abstract}
We present results for the QED contributions to the anomalous magnetic moment of the muon containing closed electron loops. The main focus is on perturbative corrections at four-loop order where the external photon couples to the external muon. Furthermore, all four-loop contributions involving simultaneously a closed electron and tau loop are computed. In combination with our recent results on the light-by-light-type corrections (see Ref. [1]) the complete four-loop electron-loop contribution to the anomalous magnetic moment of the muon has been obtained with an independent calculation. Our calculation is based on an asymptotic expansion in the ratio of the electron and the muon mass and shows the importance of higher order terms in this ratio. We perform a detailed comparison with results available in the literature and find good numerical agreement. As a by-product we present analytic results for the on-shell muon mass and wave function renormalization constants at three-loop order including massive closed electron and tau loops, which we also calculated using the method of asymptotic expansion.
\end{abstract}




\section{Introduction}

The anomalous magnetic moment of the muon, $a_{\mu}$, is an important observable in particle physics. Both on the experimental [2, 3] and the theory side a lot of effort has been invested to provide precise results for $a_{\mu}$. The theory prediction can be split into hadronic, electroweak and QED contribution. The non-perturbative hadronic contribution is further subdivided into the vacuum polarization [4-11] and light-by-light contribution [12 15] and has reached next-to-next-to-leading order accuracy. It is nevertheless the main source to the uncertainty of the theory prediction. On the other hand, the electroweak part is known up to two-loop order [16-19] and thus well under control. The numerically largest contribution arises from QED corrections. Up to three loops analytical results are available [20 29] and four and five-loop corrections have been computed in Refs. [30 32] using numerical methods. In this paper we complete the cross-check of the four-loop corrections involving at least one closed electron loop using an independent method.

We cast the perturbative expansion of $a_{\mu}$ in the form

$$
a_{\mu}=\sum_{n=1}^{\infty} a_{\mu}^{(2 n)}\left(\frac{\alpha}{\pi}\right)^{n},
$$

where $n$ counts the number of loops. It is common practice to further split the four-loop term into four parts according to

$$
a_{\mu}^{(8)}=A_{1}^{(8)}+A_{2}^{(8)}\left(m_{\mu} / m_{e}\right)+A_{2}^{(8)}\left(m_{\mu} / m_{\tau}\right)+A_{3}^{(8)}\left(m_{\mu} / m_{e}, m_{\mu} / m_{\tau}\right),
$$

where $A_{1}^{(8)}$ denotes the universal part which includes the pure photonic corrections and closed muon loops. The arguments of the remaining three contributions indicate which leptons are involved in the corresponding Feynman diagrams. Note that analytic results for $A_{2}^{(8)}\left(m_{\mu} / m_{\tau}\right)$ have been computed in Ref. [33] and the light-by-light contributions of $A_{2}^{(8)}\left(m_{\mu} / m_{e}\right)$ are presented in Ref. [1]. In this work we will concentrate on the nonlight-by-light contributions of $A_{2}^{(8)}\left(m_{\mu} / m_{e}\right)$. Furthermore, we present analytic results for $A_{3}^{(8)}\left(m_{\mu} / m_{e}, m_{\mu} / m_{\tau}\right)$. For convenience we split the last three contributions in Eq. (2) into a so-called light-by-light part and a remainder and write

$$
A_{2 / 3}^{(8)}=A_{2 / 3}^{(8), \mathrm{lbl}}+A_{2 / 3}^{(8), \mathrm{rem}}
$$

In Figure 1 we define (following Ref. [32]) a subdivision into different classes of Feynman diagrams which are individually finite and gauge invariant. All of them contribute to $A_{2}^{(8)}\left(m_{\mu} / m_{e}\right)$, those with two closed fermion loops also to $A_{3}^{(8)}\left(m_{\mu} / m_{e}, m_{\mu} / m_{\tau}\right)$. The light-by-light-type cases are denoted by $\operatorname{IV}(\mathrm{a}), \operatorname{IV}(\mathrm{b})$ and $\operatorname{IV}(\mathrm{c})$.

In contrast to $A_{2}^{(8), \mathrm{lbl}}\left(m_{\mu} / m_{e}\right)$ it is possible to obtain the leading term for $m_{e} / m_{\mu} \rightarrow 0$ of $A_{2}^{(8), r e m}\left(m_{\mu} / m_{e}\right)$ by simply setting the electron mass to zero. One obtains a finite 

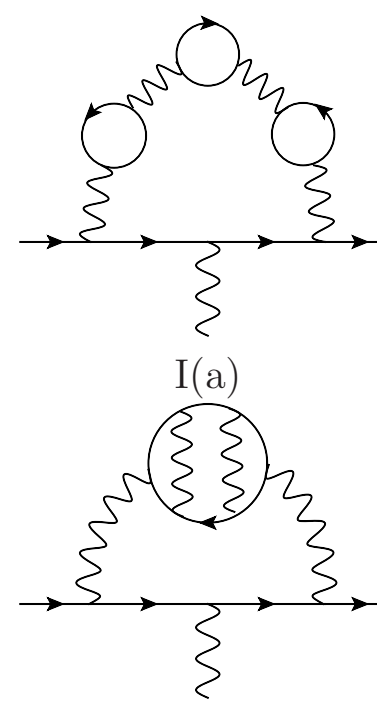

$\mathrm{I}(\mathrm{d})$

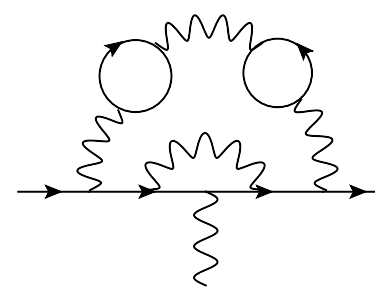

$\mathrm{II}(\mathrm{c})$

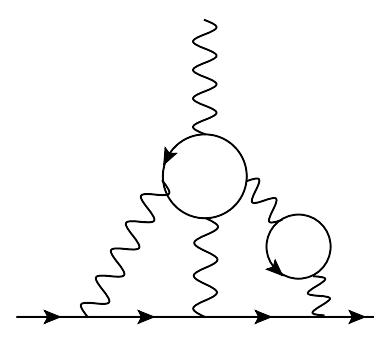

$\operatorname{IV}(\mathrm{a})$

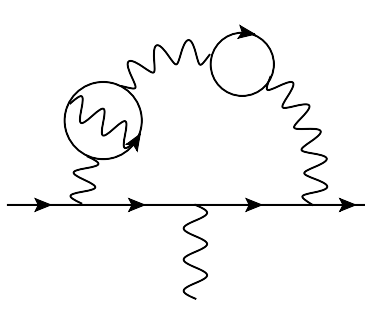

$\mathrm{I}(\mathrm{b})$

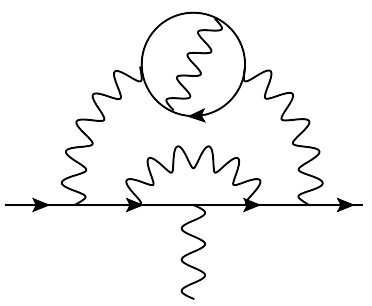

II (a)

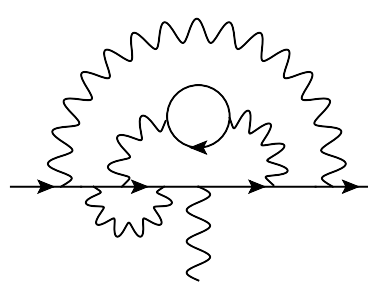

III

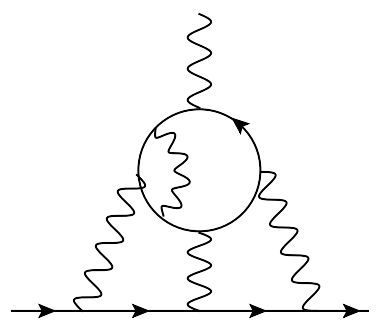

$\mathrm{IV}(\mathrm{b})$

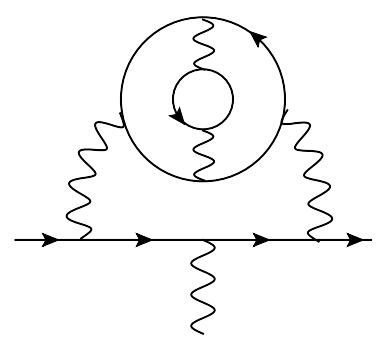

$\mathrm{I}(\mathrm{c})$
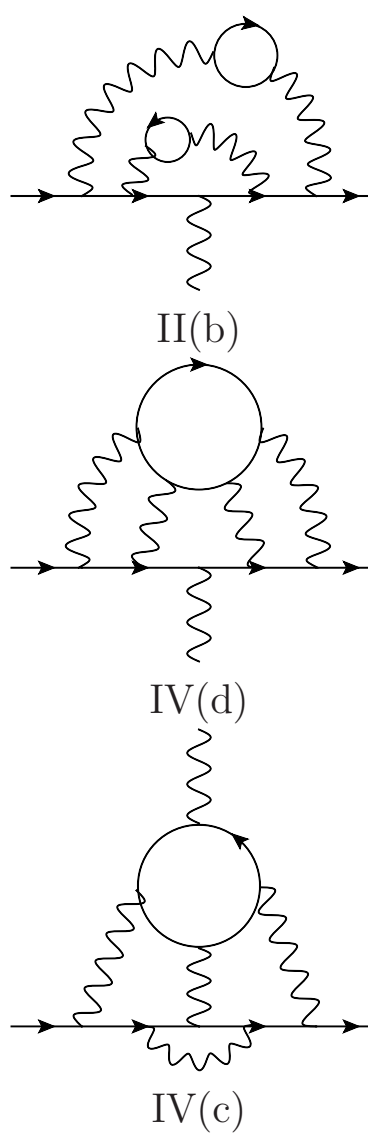

Figure 1: Four-loop example Feynman diagrams contributing to $a_{\mu}$ containing at least one closed electron loop. The external solid lines represent muons, the solid loops denote electrons, muons or taus, and the wavy lines represent photons.

result after renormalizing the coupling constant $\alpha$ in the $\overline{\mathrm{MS}}$ scheme and thus gets an expression which depends on $\log \left(\mu / m_{\mu}\right)$. Here, $\mu$ is the renormalization scale which also appear in the argument of $\bar{\alpha}(\mu)$. The transformation of $\bar{\alpha}$ to the on-shell scheme introduces $\log \left(\mu / m_{e}\right)$ terms in such a way that the renormalization scale $\mu$ drops out and a dependence on $\log \left(m_{\mu} / m_{e}\right)$ remains. This approach has been used to get analytic results for the leading term to $A_{2}^{(8), r e m}\left(m_{\mu} / m_{e}\right)$ involving two or three electron loops which 
constitute contributions to I(a), I(b), I(c), II(b) and II(c). In this paper we complement these results with higher order terms in the $m_{e} / m_{\mu}$ expansion and add numerical results for

all remaining contributions of $A_{2}^{(8), \text { rem }}\left(m_{\mu} / m_{e}\right)$. The corresponding results are presented in Section 4 .

In Section 5 we discuss the contribution $A_{3}^{(8)}\left(m_{\mu} / m_{e}, m_{\mu} / m_{\tau}\right)$, which is computed by applying a nested asymptotic expansion for the hierarchy $m_{e} \ll m_{\mu} \ll m_{\tau}$. Analytic results are presented and compared to the numerical results present in the literature.

The structure of the paper is as follows: In the next section we discuss some of the calculational challenges we met during our calculation. Afterwards we discuss in Section 3 the renormalization procedure and present new results for the three-loop renormalization constants of the muon mass and wave function which are not yet available in the literature. After presenting results for $a_{\mu}$ in Sections 4 and 5 we conclude in Section 6 . We dedicate the appendix to useful analytic results for some of the four-loop coefficients of

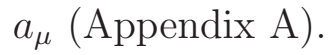

\section{Calculation details}

The integrals which have to be computed for the quantities considered in this paper are on-shell integrals where the square of the external momentum equals $m_{\mu}^{2}$. As a further scale one has the electron mass $m_{e}$ and for the corrections of Section 5 also the tau mass $m_{\tau}$ is present. Since there is a strong hierarchy among the lepton masses, $m_{e} \ll m_{\mu} \ll m_{\tau}$ we apply an asymptotic expansion to rewrite the two-scale integrals in terms of products of one-scale integrals. This procedure provides the renormalization constants and the anomalous magnetic moment in terms of a series expansion in the mass ratios. The asymptotic expansion significantly simplifies the complexity of the underlying integrals and sometimes even leads to analytic results.

We apply the strategy of expansion by regions [34 37] (see also a recent review in Chapter 9 of [38]) which provides an asymptotic expansion of a given Feynman integral in a given limit represented as a finite sum of contributions corresponding to so-called regions (i.e. scalings of components of loop momenta or Feynman parameters). Each term of such contributions is manifestly homogeneous with respect to the expansion parameter.

In Figure 2 we demonstrate the way we apply the asymptotic expansion to a typical diagram. The sub-diagrams shown in rows two to six are obtained by allowing each loop momentum to scale either as the hard scale, i.e., $m_{\mu}$, or as the soft scale which is given by the electron mass. This leads to regions which determine the expansion prescription for each propagator. For the diagram in Figure 2 one obtains five types of contributions which we discuss in the following: 
full diagram:

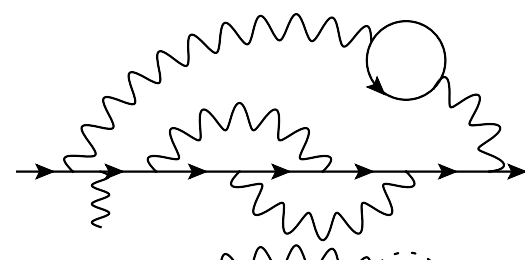

1. all-hard region:

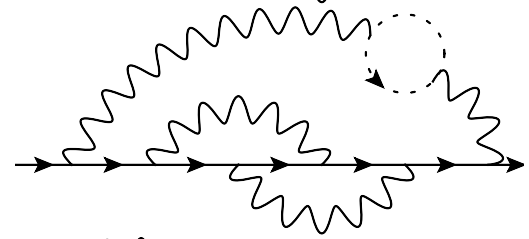

2. three hard momenta:
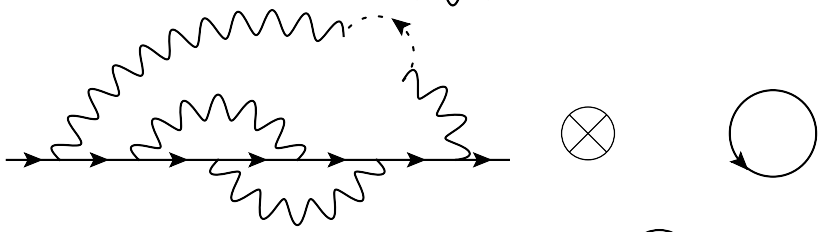

3. two hard momenta:

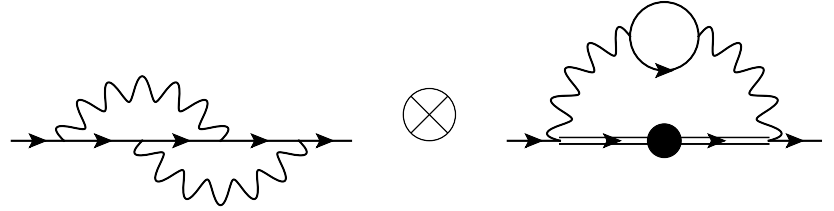

4. one hard momentum:

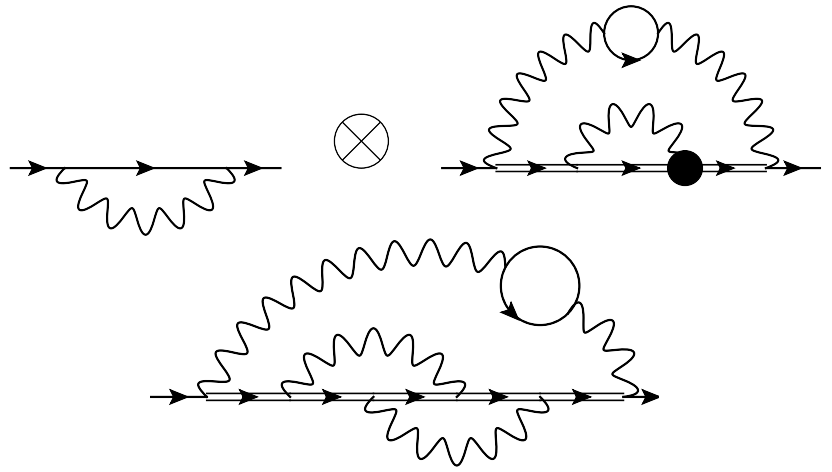

Figure 2: Relevant regions of an example Feynman diagram with an electron loop. The first line contains the full diagram and in rows two to six one representative sub-diagram for each region is shown. The dotted lines denote massless (electron) propagators, the solid lines massive (muon or electron) propagators and the solid double lines represent linear propagators of the form $1 /(2 \ell \cdot q)$ where $q$ is the external momentum with $q^{2}=m_{\mu}^{2}$ and $\ell$ is a loop momentum.

1. In case all loop momenta are hard one obtains a four-loop on-shell integral. As the electron propagators contain heavy momenta, a Taylor expansion in $m_{e}$ has to be performed and thus the electron propagators become massless.

2. There are two contributions corresponding to regions where one of the electron propagators is soft and the momenta through all other propagators are hard. This situation is obtained in case the loop momentum in the electron loop is of order 
$\ell_{1} \sim m_{e}$ and none of the other momenta are flowing through one of the electron propagators. This propagator leads to a one-loop vacuum integral with mass $m_{e}$. The other part of the diagram becomes a three-loop on-shell integral. Note that the second electron propagator has to be expanded in $\ell_{1}$ and $m_{e}$ which looks as follows

$$
\frac{1}{\left(\ell_{1}+\ell_{2}\right)^{2}-m_{e}^{2}}=\frac{1}{\ell_{2}^{2}} \sum_{n=0}^{\infty}\left(\frac{-\ell_{1}^{2}-2 \ell_{1} \cdot \ell_{2}+m_{e}^{2}}{\ell_{2}^{2}}\right)^{n} .
$$

3. A further contribution corresponds to the region, where the two loop momenta through the photon lines which are not connected to the electron loop are hard. This leads to a two-loop on-shell integral. In the remaining two-loop integral where both loop momenta scale like $m_{e}$ one has to choose the external momentum along the muon lines. The corresponding propagators have to be expanded according to $\left(\ell_{1} \sim m_{e}\right)$

$$
\frac{1}{\left(\ell_{1}+q\right)^{2}-m_{\mu}^{2}}=\frac{1}{\ell_{1}^{2}+2 \ell_{1} \cdot q}=\frac{1}{2 \ell_{1} \cdot q} \sum_{n=0}^{\infty}\left(\frac{-\ell_{1}^{2}}{2 \ell_{1} \cdot q}\right)^{n} .
$$

The linear propagator $1 /\left(2 \ell_{1} \cdot q\right)^{n}$ does not introduce an additional scale, because it only gives rise to an overall factor $\left(q^{2}\right)^{-n / 2}$. The rest of the integral is independent of $q^{2}$ and also $m_{\mu}$.

4. If one replaces in the previous region one of the hard loop momenta by a soft one, one obtains a one-loop on-shell integral and a three-loop linear integral.

5. In the last region all loop momenta are of order $m_{e}$ and the external muon momentum must again flow through the muon line. This leads to a four-loop integral which has only the scale $m_{e} ; m_{\mu}$ only appears as a trivial pre-factor.

The relevant regions can be found by examining the scaling behaviour of the alphaparameter representation of a given Feynman diagram [35. This approach is implemented in the Mathematica package asy [36,37]. An alternative approach, which is implemented in an in-house Mathematica program, is based on the fact that each loop momentum is either hard or soft. More details are given in Ref. [1] where the light-by-light-type four-loop contribution has been computed.

Let us mention that a non-trivial issue is the calculation of the tensor integrals which occur due to the factorization of the integrals. The most complicated cases which we had to implement for our calculation were tensor integrals up to rank ten for three-loop vacuum integrals and up to rank ten and eight for one- and two-loop on-shell integrals, respectively.

The reduction of the tensor structure as well as the evaluation of traces of $\gamma$-matrices is done with FORM [39, 40] and TFORM [41] (see also Ref. [42]). The scalar integrals are 
reduced with FIRE [43] and crusher [44] to a relative small set of master integrals, 1] Some of them could be evaluated analytically or to high numerical precision. The $\epsilon$ expansion of the remaining ones is computed numerically with the help of FIESTA [46]. Further details can be found in Ref. [1. Let us stress that our approach leads to an analytic expression for $a_{\mu}$ as a linear combination of master integrals. Thus, if necessary, the accuracy of the final result can be systematically improved.

\section{On-shell renormalization constants}

In this section we discuss the renormalization procedure and provide results for the threeloop renormalization constants which are not yet available in the literature.

The four-loop calculation performed in this paper requires the on-shell renormalization of the muon mass and wave function to three-loop order. We adopt the same notation as for $a_{\mu}$ and define

$$
Z_{2 / m}^{\mathrm{OS}}=Z_{2 / m, 1}^{\mathrm{OS}}+\delta Z_{2 / m, 2}^{\mathrm{OS}}\left(m_{\mu} / m_{e}\right)+\delta Z_{2 / m, 2}^{\mathrm{OS}}\left(m_{\mu} / m_{\tau}\right)+\delta Z_{2 / m, 3}^{\mathrm{OS}}\left(m_{\mu} / m_{e}, m_{\mu} / m_{\tau}\right) .
$$

Up to two loops the mass and wave function renormalization constant can be found in Refs. [47,48]. At three-loop order only the limit $m_{e} \rightarrow 0$ exists in analytic form [49 51] (see also Refs. [52, 53] where $Z_{m}^{\mathrm{OS}}$ has been computed for the first time using numerical methods) whereas the fermionic corrections are only known in numerical form [54].2 In this section we present analytic results for $\delta Z_{2 / m, 2}^{\mathrm{OS}}\left(m_{\mu} / m_{e}\right)$ including correction terms in $m_{e} / m_{\mu}$. They are needed to construct the result for $a_{\mu}$ shown in Section 4. Furthermore, we also present the results for $\delta Z_{2 / m, 3}^{\mathrm{OS}}\left(m_{\mu} / m_{e}, m_{\mu} / m_{\tau}\right)$ which are needed for the construction of $a_{\mu}$ from Section 5. The results for $\delta Z_{2 / m, 2}^{\mathrm{OS}}\left(m_{\mu} / m_{\tau}\right)$ can be found in Ref. [33].

Note that the renormalization of the electron mass is needed up to two loops. The corresponding counterterm can be obtained from Refs. [47,54].

As a further ingredient the on-shell renormalization constant of $\alpha$ is needed up to three loops. In the practical calculation we renormalize the coupling in a first step in the $\overline{\mathrm{MS}}$ scheme and transform afterwards to the on-shell scheme. The corresponding conversion formula with massive leptons can be obtained from Ref. [56].

Sample diagrams which contribute to $Z_{m}^{\mathrm{OS}}$ and $Z_{2}^{\mathrm{OS}}$ are shown in Figure 3 , For the

\footnotetext{
${ }^{1}$ The four-loop on-shell master integrals are a subset of the ones which are needed for the quark mass relation considered in Ref. 45. In addition there are 128 four-loop "linear masters" with at least one propagator of the form $1 /(2 \ell \cdot q)$.

${ }^{2}$ Analytic, asymptotically expanded three-loop results for the light-fermion contribution to $Z_{2}^{\mathrm{OS}}$ can be found in Ref. 55.
} 

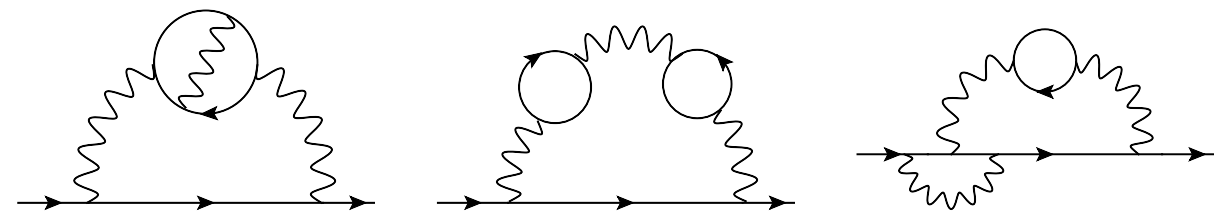

Figure 3: Propagator type diagrams with electron loops contributing to the mass and field renormalization constants. The middle diagram can contain two electron loops or one electron and one muon loop.

computation of the corresponding integrals we apply the asymptotic expansion described in the previous section and obtain the result as a series in $m_{e} / m_{\mu}$.

In the following we present the three-loop electron contributions to $Z_{m}^{\mathrm{OS}}$ and $Z_{2}^{\mathrm{OS}}$ parametrized in terms of the fine structure constant in the $\overline{\mathrm{MS}}$ scheme, $\bar{\alpha}$. The masses $m_{e}$ and $m_{\mu}$ are renormalized on-shell. The symbols $\ell_{i}$ with $i=e, \mu$ denote $\log \left(\mu^{2} / m_{i}^{2}\right)$, where $\mu$ is the renormalization scale. Moreover we label closed electron and muon loops by $n_{e}$ and $n_{\mu}$, respectively. Furthermore, $\zeta_{j}$ denotes Riemann's zeta function and $a_{4}=\operatorname{Li}_{4}(1 / 2)$. Our results for three-loop contributions to $\delta Z_{m, 2}^{\mathrm{OS}}\left(m_{\mu} / m_{e}\right)$ and $\delta Z_{2,2}^{\mathrm{OS}}\left(m_{\mu} / m_{e}\right)$ read

$$
\begin{aligned}
& \delta^{(3)} Z_{m, 2}^{\mathrm{OS}}=\left(\frac{\bar{\alpha}}{\pi}\right)^{3}\left(n _ { e } n _ { \mu } \left[-\frac{1}{18 \epsilon^{3}}+\frac{5}{108 \epsilon^{2}}+\frac{35}{648 \epsilon}-\frac{\ell_{\mu}^{3}}{18}-\frac{13 \ell_{\mu}^{2}}{36}+\frac{13 \pi^{2}}{108}\right.\right. \\
& +\left(\frac{\pi^{2}}{18}-\frac{143}{108}\right) \ell_{\mu}+\frac{2 \zeta_{3}}{9}-\frac{5917}{3888}+\frac{\pi^{2} \ell_{\mu} m_{e}}{6 m_{\mu}}+\frac{m_{e}^{2}}{m_{\mu}^{2}}\left(-\ell_{\mu}-\frac{2}{3}\right) \\
& \left.+\frac{m_{e}^{3}}{m_{\mu}^{3}}\left(\frac{\ell_{\mu} \pi^{2}}{6}+\frac{4 \pi^{2}}{45}\right)\right]+n_{e}\left[\frac{3}{32 \epsilon^{3}}+\frac{1}{\epsilon^{2}}\left(\frac{3 \ell_{\mu}}{32}-\frac{5}{192}\right)+\frac{1}{\epsilon}\left(-\frac{3 \ell_{\mu}^{2}}{64}\right.\right. \\
& \left.-\frac{7 \pi^{2}}{128}-\frac{23 \ell_{\mu}}{64}-\frac{\zeta_{3}}{4}-\frac{65}{384}+\frac{3 \pi^{2} m_{e}}{16 m_{\mu}}-\frac{9 m_{e}^{2}}{8 m_{\mu}^{2}}+\frac{3 \pi^{2} m_{e}^{3}}{16 m_{\mu}^{3}}\right) \\
& +\ell_{\mu}\left(-\frac{\pi^{2} \log (2)}{3}+\frac{5 \pi^{2}}{384}-\frac{\zeta_{3}}{4}-\frac{497}{384}\right)-\frac{11 \ell_{\mu}^{3}}{64}-\frac{117 \ell_{\mu}^{2}}{128}+\frac{\log ^{4}(2)}{9} \\
& +\frac{2 \pi^{2} \log ^{2}(2)}{9}-\frac{11 \pi^{2} \log (2)}{9}-\frac{119 \pi^{4}}{2160}+\frac{1091 \pi^{2}}{2304}+\frac{8 a_{4}}{3}+\frac{145 \zeta_{3}}{96} \\
& +\frac{575}{2304}+\frac{m_{e}}{m_{\mu}}\left(\frac{13 \pi^{3}}{18}+\frac{3 \ell_{e} \pi^{2}}{8}+\frac{3 \ell_{\mu} \pi^{2}}{16}+\frac{5 \pi^{2} \log (2)}{18}-\frac{751 \pi^{2}}{432}\right) \\
& +\frac{m_{e}^{2}}{m_{\mu}^{2}}\left(\frac{3 \pi^{2}}{4}-\frac{9 \ell_{e}}{4}-\frac{9 \ell_{\mu}}{8}-\frac{51}{8}\right)+\frac{m_{e}^{3}}{m_{\mu}^{3}}\left(\frac{7 \pi^{3}}{12}+\frac{11 \ell_{e} \pi^{2}}{72}+\frac{59 \ell_{\mu} \pi^{2}}{144}\right. \\
& \left.\left.+\frac{5 \pi^{2} \log (2)}{18}-\frac{49 \pi^{2}}{36}\right)\right]+n_{e}^{2}\left[-\frac{1}{36 \epsilon^{3}}+\frac{5}{216 \epsilon^{2}}+\frac{35}{1296 \epsilon}-\frac{\ell_{\mu}^{3}}{36}\right. \\
& -\frac{13 \ell_{\mu}^{2}}{72}+\left(-\frac{\pi^{2}}{18}-\frac{89}{216}\right) \ell_{\mu}-\frac{13 \pi^{2}}{108}-\frac{7 \zeta_{3}}{18}-\frac{2353}{7776}
\end{aligned}
$$




$$
\begin{aligned}
& \left.\left.+\frac{m_{e}}{m_{\mu}}\left(\frac{\ell_{e} \pi^{2}}{6}+\frac{4 \pi^{2}}{45}\right)+\frac{m_{e}^{2}}{m_{\mu}^{2}}\left(-\ell_{\mu}-\frac{2}{3}\right)+\frac{\ell_{e} \pi^{2} m_{e}^{3}}{6 m_{\mu}^{3}}\right]+\mathcal{O}\left(\frac{m_{e}^{4}}{m_{\mu}^{4}}\right)\right), \\
& \delta^{(3)} Z_{2,2}^{\mathrm{OS}}=\left(\frac{\bar{\alpha}}{\pi}\right)^{3}\left(n _ { e } n _ { \mu } \left[\frac{1}{36 \epsilon^{2}}+\frac{1}{\epsilon}\left(-\frac{\ell_{e} \ell_{\mu}}{6}-\frac{5}{216}\right)-\frac{\ell_{\mu}^{3}}{12}-\frac{19 \ell_{\mu}^{2}}{36}-\frac{\ell_{e}^{2} \ell_{\mu}}{6}\right.\right. \\
& +\left(-\frac{355}{108}+\frac{11 \pi^{2}}{72}\right) \ell_{\mu}+\ell_{e}\left(-\frac{\ell_{\mu}^{2}}{12}+\frac{2 \ell_{\mu}}{9}-\frac{\pi^{2}}{72}\right)+\frac{13 \pi^{2}}{36}-\frac{4721}{1296} \\
& \left.+\frac{\pi^{2} \ell_{\mu} m_{e}}{4 m_{\mu}}+\frac{m_{e}^{2}}{m_{\mu}^{2}}\left(\frac{\ell_{e}}{5}-\frac{11 \ell_{\mu}}{5}-\frac{13}{25}\right)+\frac{m_{e}^{3}}{m_{\mu}^{3}}\left(\frac{5 \pi^{2} \ell_{\mu}}{12}+\frac{2 \pi^{2}}{15}\right)\right] \\
& +n_{e}\left[\frac{1}{\epsilon^{2}}\left(-\frac{3 \ell_{e}}{16}-\frac{7}{192}\right)+\frac{1}{\epsilon}\left(-\frac{3 \ell_{e}^{2}}{16}-\frac{3 \ell_{\mu}^{2}}{32}+\ell_{e}\left(\frac{3}{16}-\frac{3 \ell_{\mu}}{16}\right)\right.\right. \\
& \left.-\frac{41 \ell_{\mu}}{64}-\frac{5 \pi^{2}}{64}-\frac{203}{384}+\frac{9 \pi^{2} m_{e}}{32 m_{\mu}}-\frac{9 m_{e}^{2}}{4 m_{\mu}^{2}}+\frac{15 \pi^{2} m_{e}^{3}}{32 m_{\mu}^{3}}\right)-\frac{\ell_{e}^{3}}{8}-\frac{3 \ell_{\mu}^{3}}{16} \\
& +\left(\frac{9}{32}-\frac{3 \ell_{\mu}}{16}\right) \ell_{e}^{2}-\frac{179 \ell_{\mu}^{2}}{128}+\left(-\frac{3 \ell_{\mu}^{2}}{32}+\frac{3 \zeta_{3}}{4}-\frac{3 \pi^{2}}{64}+\frac{29}{48}\right) \ell_{e}+\frac{28 a_{4}}{3} \\
& +\frac{641 \zeta_{3}}{96}+\frac{7 \log ^{4}(2)}{18}+\ell_{\mu}\left(\frac{\zeta_{3}}{4}-\frac{2}{3} \pi^{2} \log (2)+\frac{31 \pi^{2}}{96}-\frac{537}{128}\right) \\
& +\frac{5}{18} \pi^{2} \log ^{2}(2)-\frac{47}{18} \pi^{2} \log (2)-\frac{59 \pi^{4}}{432}+\frac{2881 \pi^{2}}{2304}-\frac{5875}{2304} \\
& +\frac{m_{e}}{m_{\mu}}\left(\frac{9 \pi^{2} \ell_{e}}{16}+\frac{5}{12} \pi^{2} \log (2)+\frac{13 \pi^{3}}{12}+\frac{9 \ell_{\mu} \pi^{2}}{32}-\frac{751 \pi^{2}}{288}\right) \\
& +\frac{m_{e}^{2}}{m_{\mu}^{2}}\left(\left(\frac{\ell_{\mu}}{2}-\frac{\ell_{e}^{2}}{4}-\frac{67}{12}\right) \ell_{e}-\frac{\ell_{\mu}^{2}}{4}-\frac{7 \ell_{\mu}}{6}+\frac{17 \pi^{2}}{8}-\frac{1267}{72}\right) \\
& \left.+\frac{m_{e}^{3}}{m_{\mu}^{3}}\left(\frac{47 \pi^{2} \ell_{e}}{144}+\frac{41}{36} \pi^{2} \log (2)+\frac{35 \pi^{3}}{24}+\frac{311 \ell_{\mu} \pi^{2}}{288}-\frac{47 \pi^{2}}{12}\right)\right] \\
& +n_{e}^{2}\left[\frac{1}{72 \epsilon^{2}}+\frac{1}{\epsilon}\left(-\frac{\ell_{e}^{2}}{12}-\frac{5}{432}\right)-\frac{5 \ell_{e}^{3}}{36}+\frac{\ell_{e}^{2}}{9}-\frac{\ell_{\mu}^{3}}{36}-\frac{19 \ell_{\mu}^{2}}{72}\right. \\
& +\left(-\frac{31}{108}-\frac{\pi^{2}}{72}\right) \ell_{e}+\ell_{\mu}\left(-\frac{167}{216}-\frac{\pi^{2}}{18}\right)-\frac{19 \pi^{2}}{108}-\frac{2449}{2592} \\
& \left.\left.+\frac{m_{e}}{m_{\mu}}\left(\frac{\pi^{2} \ell_{e}}{4}+\frac{2 \pi^{2}}{15}\right)+\frac{m_{e}^{2}}{m_{\mu}^{2}}\left(-2 \ell_{\mu}-\frac{7}{3}\right)+\frac{5 \pi^{2} \ell_{e} m_{e}^{3}}{12 m_{\mu}^{3}}\right]+\mathcal{O}\left(\frac{m_{e}^{4}}{m_{\mu}^{4}}\right)\right) \text {. }
\end{aligned}
$$

$\delta^{(3)} Z_{m, 2}^{\mathrm{OS}}$ and $\delta^{(3)} Z_{2,2}^{\mathrm{OS}}$ agree with the numerical results given in Ref. [54] and $\delta^{(3)} Z_{2,2}^{\mathrm{OS}}$ agrees with the analytic expression of Ref. [55].

For $\delta Z_{m, 3}^{\mathrm{OS}}\left(m_{\mu} / m_{e}, m_{\mu} / m_{\tau}\right)$ and $\delta Z_{2,3}^{\mathrm{OS}}\left(m_{\mu} / m_{e}, m_{\mu} / m_{\tau}\right)$ we obtain

$$
\delta Z_{m, 3}^{\mathrm{OS}}=\left(\frac{\bar{\alpha}}{\pi}\right)^{3} n_{e} n_{\tau}\left[-\frac{1}{18 \epsilon^{3}}+\frac{5}{108 \epsilon^{2}}+\frac{35}{648 \epsilon}+\frac{\ell_{\tau}^{3}}{36}+\ell_{\tau}\left(-\frac{1}{8}-\frac{13 \ell_{\mu}}{36}-\frac{\ell_{\mu}^{2}}{12}-\frac{\pi^{2}}{18}\right)\right.
$$




$$
\begin{aligned}
& -\frac{1327}{3888}+\frac{2 \zeta_{3}}{9}+\frac{\ell_{\tau} \pi^{2}}{6} \frac{m_{e}}{m_{\mu}}-\frac{\ell_{\tau} m_{e}^{2}}{m_{\mu}^{2}}+\left(-\frac{46}{75}-\frac{\ell_{\mu}}{5}+\frac{\ell_{\tau}}{5}\right) \frac{m_{e}^{2}}{m_{\tau}^{2}} \\
& +\left(\frac{529}{3375}-\frac{2 \ell_{\mu}}{45}-\frac{\ell_{\mu}^{2}}{45}-\frac{\ell_{\tau}}{25}+\frac{\ell_{\tau}^{2}}{45}-\frac{2 \pi^{2}}{135}\right) \frac{m_{\mu}^{2}}{m_{\tau}^{2}} \\
& +\frac{\ell_{\tau} \pi^{2}}{6} \frac{m_{e}^{3}}{m_{\mu}^{3}}+\frac{4 \pi^{2} m_{e}^{3}}{45 m_{\mu} m_{\tau}^{2}}+\left(-\frac{142}{3675}-\frac{\ell_{\mu}}{35}+\frac{\ell_{\tau}}{35}\right) \frac{m_{e}^{2} m_{\mu}^{2}}{m_{\tau}^{4}} \\
& +\left(\frac{552889}{16464000}-\frac{9 \ell_{\mu}}{1120}-\frac{3 \ell_{\mu}^{2}}{560}-\frac{37 \ell_{\tau}}{9800}+\frac{3 \ell_{\tau}^{2}}{560}-\frac{\pi^{2}}{280}\right) \frac{m_{\mu}^{4}}{m_{\tau}^{4}} \\
& +\left(\frac{99227}{7501410}-\frac{38 \ell_{\mu}}{14175}-\frac{2 \ell_{\mu}^{2}}{945}-\frac{199 \ell_{\tau}}{297675}+\frac{2 \ell_{\tau}^{2}}{945}-\frac{4 \pi^{2}}{2835}\right) \frac{m_{\mu}^{6}}{m_{\tau}^{6}} \\
& \left.+\left(-\frac{286}{33075}-\frac{\ell_{\mu}}{105}+\frac{\ell_{\tau}}{105}\right) \frac{m_{e}^{2} m_{\mu}^{4}}{m_{\tau}^{6}}+\ldots\right] \text {, } \\
& \delta Z_{2,3}^{\mathrm{OS}}=\left(\frac{\bar{\alpha}}{\pi}\right)^{3} n_{e} n_{\tau}\left[\frac{1}{36 \epsilon^{2}}-\frac{5}{216 \epsilon}-\frac{\ell_{e} \ell_{\tau}}{6 \epsilon}-\frac{\ell_{e}^{2} \ell_{\tau}}{6}-\frac{35}{1296}+\ell_{e}\left(\frac{2 \ell_{\tau}}{9}-\frac{\ell_{\tau}^{2}}{12}-\frac{\pi^{2}}{72}\right)\right. \\
& +\ell_{\tau}\left(-\frac{229}{216}-\frac{19 \ell_{\mu}}{36}-\frac{\ell_{\mu}^{2}}{12}-\frac{5 \pi^{2}}{72}\right)+\frac{\ell_{\tau} \pi^{2}}{4} \frac{m_{e}}{m_{\mu}}-\frac{2 \ell_{\tau} m_{e}^{2}}{m_{\mu}^{2}} \\
& +\left(-\frac{2}{45}-\frac{2 \ell_{\mu}}{45}\right) \frac{m_{\mu}^{2}}{m_{\tau}^{2}}+\left(-\frac{1}{2}+\frac{\ell_{e}}{5}-\frac{\ell_{\mu}}{5}\right) \frac{m_{e}^{2}}{m_{\tau}^{2}}+\frac{5 \ell_{\tau} \pi^{2}}{12} \frac{m_{e}^{3}}{m_{\mu}^{3}} \\
& +\frac{2 \pi^{2}}{15} \frac{m_{e}^{3}}{m_{\mu} m_{\tau}^{2}}-\frac{m_{e}^{2} m_{\mu}^{2}}{35 m_{\tau}^{4}}+\left(-\frac{685189}{16464000}-\frac{3 \ell_{\mu}}{1120}+\frac{3 \ell_{\mu}^{2}}{560}\right. \\
& \left.+\frac{37 \ell_{\tau}}{9800}-\frac{3 \ell_{\tau}^{2}}{560}+\frac{\pi^{2}}{280}\right) \frac{m_{\mu}^{4}}{m_{\tau}^{4}}+\left(-\frac{29}{33075}+\frac{\ell_{\mu}}{105}-\frac{\ell_{\tau}}{105}\right) \frac{m_{e}^{2} m_{\mu}^{4}}{m_{\tau}^{6}} \\
& \left.+\left(\frac{16 \ell_{\mu}}{14175}+\frac{4 \ell_{\mu}^{2}}{945}+\frac{398 \ell_{\tau}}{297675}-\frac{4 \ell_{\tau}^{2}}{945}+\frac{8 \pi^{2}}{2835}-\frac{546409}{18753525}\right) \frac{m_{\mu}^{6}}{m_{\tau}^{6}}+\ldots\right],
\end{aligned}
$$

where the ellipses stand for higher orders in $1 / m_{\tau}$ and $m_{e}$.

\section{Electron contribution to $a_{\mu}$}

In this section we present results for the four-loop contribution $A_{2}^{(8)}\left(m_{\mu} / m_{e}\right)$ which involves one or more closed electron loops. The application of asymptotic expansion leads to a series expansion in the parameter $x=m_{e} / m_{\mu}=1 / 206.76 \ldots$. Since $x \ll 1$ the convergence of the expansion is in general quite fast as we demonstrate by evaluating the first four expansion terms. In general it is necessary to include the linear term in $x$ to

obtain a result with sub-percent accuracy. On the other hand, the term of order $x^{3}$ are negligible in all cases. 
In the following we discuss in detail all diagram classes (cf. Figure 1) contributing to $A_{2}^{(8), \text { rem }}\left(m_{\mu} / m_{e}\right)$; the light-by-light-type results can be found in Ref. [1].

We illustrate for each class how the numerical values of the final results are build up from the expansion terms in $x \approx 1 / 206.7682843(52)$ and the logarithmic contributions. First the expansion in $x$ and its dependence on $\ell_{x}=\log (x)$ is shown. After the first equality sign the numerical values of $x$ and $\ell_{x} \approx-5.33 \ldots$ are inserted, but the resulting summands are kept separated, which indicates the relative behaviour between the constant and the logarithmic terms. Afterwards the sums for every order in $x$ are evaluated, so the convergence of the asymptotic series is demonstrated. At the end the final contribution of the diagram class is shown.

In the course of the numerical evaluation of $a_{\mu}$ there are several sources for numerical uncertainties which originate from numerical Monte-Carlo integrations. We interpret them as statistical one-sigma uncertainties and combine them in quadrature to arrive at the final uncertainty.

Diagram class I(a) consists of diagrams with three fermion loop insertions in one photon line. According to the type of the internal fermions we split this class further into I(a0), $\mathrm{I}(\mathrm{a} 1)$ and $\mathrm{I}(\mathrm{a} 2)$ where in the case of $\mathrm{I}(\mathrm{a} 0)$ all fermion loops are electrons, the subclass I(a1) has one muon loop and two electron loops, and in the case of I(a2) two of the loops consist of muons and just one is an electron loop. The results for the three sub-classes read

$$
\begin{aligned}
& A_{2}^{(8) \mathrm{I}(\mathrm{a} 0)}=-3.266377-2.687872 \ell_{x}-0.925926 \ell_{x}^{2}-0.148148 \ell_{x}^{3} \\
& +x[6.40516] \\
& +x^{2}\left[17.24475+20.03224 \ell_{x}+5.77778 \ell_{x}^{2}+1.77778 \ell_{x}^{3}\right] \\
& +x^{3}[-52.0022] \\
& =[-3.26638+14.33065-26.32032+22.45270] \\
& +[0.0309775] \\
& +[0.000403-0.002498+0.003842-0.006302] \\
& +[-0.000005883] \\
& =[7.19666]+[0.03098]+[-0.004555]+[-0.000005883] \\
& =7.22308 \text {, } \\
& A_{2}^{(8), \mathrm{I}(\mathrm{a} 1)}=0.0167998+0.0220046 \ell_{x}+0.0209166 \ell_{x}^{2} \\
& +x[0] \\
& +x^{2}\left[-0.548361-0.254463 \ell_{x}\right] \\
& +x^{3}[0] \\
& =[0.016700-0.117320+0.594573]+[0] \\
& +[-0.00001283+0.00003173]+[0] \\
& =[0.494053]+[0]+[0.00001891]+[0]
\end{aligned}
$$




$$
\begin{aligned}
= & 0.494072 \\
A_{2}^{(8), \mathrm{I}(\mathrm{a} 2)}= & 0.000706151-0.00511705 \ell_{x} \\
& +x[0] \\
& +x^{2}[0.00493387] \\
& +x^{3}[0] \\
= & {[0.0007062+0.0272821]+[0] } \\
& +[0.0000001154]+[0] \\
= & {[0.0279882]+[0]+[0.0000001154]+[0] } \\
= & 0.0279883 .
\end{aligned}
$$

Due to the three electron loops the $x^{0}$ term of I(a0) has a cubic logarithmic correction. One observes sizeable cancellations among the individual logarithmic contributions which results in a final contribution of about 7.2. Note that the odd powers in $x$ have no logarithmic enhancement terms. As a result, the higher order corrections in $x$ lead to a shift at the sub-percent level. The contributions with one or two muon loops are numerically less important. On the one hand this is connected to the lower maximal logarithmic power of the leading order term. On the other hand one also observes that the odd powers of $x$ have vanishing coefficients. Note that for the cases I(a1) and I(a2) the approximation of a massless electron is very precise.

The diagram classes I(b) and I(c) are constructed from the one-loop diagram by adding either a two-loop and a one-loop fermion insertion or a three-loop double-bubble diagram. We refrain from separating the corresponding counterterm contribution for $\alpha$ and thus we consider the sum of $\mathrm{I}(\mathrm{b})$ and $\mathrm{I}(\mathrm{c})$ which we denote by $\mathrm{I}(\mathrm{bc})$. In analogy to case $\mathrm{I}(\mathrm{a})$ the case $\mathrm{I}(\mathrm{bc})$ is split into a contribution with two electron loops $[\mathrm{I}(\mathrm{bc} 0)]$ and contributions with an electron and a muon loop $[\mathrm{I}(\mathrm{bc} 1)$ and $\mathrm{I}(\mathrm{bc} 2)]$. In case of $\mathrm{I}(\mathrm{bc} 1)$ the electron loop has four photon couplings, in $\mathrm{I}(\mathrm{bc} 2)$ it is the muon loop. Our results read

$$
\begin{aligned}
A_{2}^{(8), \mathrm{I}(\mathrm{bc} 0)}= & 0.558875+0.714610 \ell_{x}+0.416667 \ell_{x}^{2} \\
& +x[-8.77188 \pm 0.00035] \\
& +x^{2}\left[-51.3498-61.6586 \ell_{x}-18 \ell_{x}^{2}-6.6667 \ell_{x}^{3}\right] \\
& +x^{3}[176.8096 \pm 0.0014] \\
= & 0.55887-3.81001+11.84414] \\
& +[-0.0424237 \pm 0.0000017] \\
& +[-0.00120+0.00769-0.01197+0.02363] \\
& +[0.00002000] \\
= & {[8.59301] } \\
& +[-0.0424237 \pm 0.0000017] \\
& +[0.018153] \\
& +[0.00002000]
\end{aligned}
$$




$$
\begin{aligned}
& =8.568755 \pm 0.000002, \\
& A_{2}^{(8), \mathrm{I}(\mathrm{bc} 1)}=0.057516 \pm 0.001200-0.0156874 \ell_{x} \\
& +x[0] \\
& +x^{2}\left[0.30059 \pm 0.00017+0.359473 \ell_{x}\right] \\
& +x^{3}\left[3.15081-0.292433 \ell_{x}\right] \\
& =[0.057516 \pm 0.001200+0.083639] \\
& +[0] \\
& +[0.0000070307 \pm 0.000000040-0.0000448286] \\
& +[0.0000003564+0.0000001764] \\
& =[0.141155 \pm 0.001200] \\
& +[0] \\
& +[-0.0000377979 \pm 0.0000000040] \\
& +[0.0000005328] \\
& =0.1411 \pm 0.0012 \text {, } \\
& A_{2}^{(8), \mathrm{I}(\mathrm{bc} 2)}=-0.068531 \pm 0.000075-0.105741 \ell_{x} \\
& +x[0.0626838] \\
& +x^{2}[0.422851 \pm 0.000053] \\
& +x^{3}[-3.26780] \\
& =[-0.068531 \pm 0.000075+0.563770] \\
& +[0.0003032] \\
& +[0.0000098905 \pm 0.0000000012] \\
& +[-0.0000003697] \\
& =[0.495239 \pm 0.000075] \\
& +[0.0003032] \\
& +[0.0000098905 \pm 0.0000000012] \\
& +[-0.0000003697] \\
& =0.495552 \pm 0.000075 \text {. }
\end{aligned}
$$

Similarly to the subsets of I(a) the diagram class $\mathrm{I}(\mathrm{bc} 0)$ is more than one order of magnitude larger compared to $\mathrm{I}(\mathrm{bc} 1)$ and $\mathrm{I}(\mathrm{bc} 2)$. The higher order corrections of $\mathrm{I}(\mathrm{bc} 0)$ is below one per cent and in case of $\mathrm{I}(\mathrm{bc} 1)$ and $\mathrm{I}(\mathrm{bc} 2)$ even smaller. It is interesting to note that the $x^{2}$ contribution of $\mathrm{I}(\mathrm{bc} 0)$ is around $40 \%$ of the size of the $x^{1}$ part, though the order $x^{3}$ term is much smaller justifying the truncation of the expansion.

Our result for diagram class $\mathrm{I}(\mathrm{d})$ reads

$$
\begin{aligned}
A_{2}^{(8), \mathrm{I}(\mathrm{d})}= & -0.124375+0.03125 \ell_{x} \\
& +x[17.4222 \pm 0.0997]
\end{aligned}
$$




$$
\begin{aligned}
& +x^{2}\left[40.1185+53.5293 \ell_{x}+5.25 \ell_{x}^{2}+6 \ell_{x}^{3}\right] \\
& +x^{3}[-349.570 \pm 2.148] \\
= & {[-0.124375-0.166612] } \\
& +[0.08426 \pm 0.00048] \\
& +[0.0009384-0.0066755+0.0034907-0.0212694] \\
& +[-0.00003954 \pm 0.00000024] \\
= & -0.290987] \\
& +[0.08426 \pm 0.00048] \\
& +[-0.0235159] \\
& +[-0.00003954 \pm 0.00000024] \\
= & -0.23028 \pm 0.00048 .
\end{aligned}
$$

This contribution is an example where the first terms of the asymptotic expansion provides a bad approximation to the final result. In fact, the linear term is about $30 \%$ of the leading order contribution. Also the quadratic correction is only about a factor four smaller than the linear term. However, the $x^{3}$ term is at per mille level and justifies the assignment of a negligible uncertainty from the asymptotic expansion.

The diagram classes II(a), II(b) and II(c) can be constructed from the two-loop diagrams with two photons, where either a two-loop electron self energy is inserted in one of the photon lines $[\mathrm{II}(\mathrm{a})]$, a one-loop contribution is inserted in each photon line $[\mathrm{II}(\mathrm{b})]$ or two one-loop contribution are inserted in one of the photons $[\mathrm{II}(\mathrm{c})]$. We combine again the contributions from $\mathrm{II}(\mathrm{b})$ and $\mathrm{II}(\mathrm{c})$ and denote by $\mathrm{II}(\mathrm{bc} 0)$ and $\mathrm{II}(\mathrm{bc} 1)$ the cases with two electron loops and one electron and one muon loop, respectively. Our results are given by

$$
\begin{aligned}
A_{2}^{(8), \mathrm{II}(\mathrm{a})}= & -0.934278+0.344166 \ell_{x} \\
& +x[-2.83881] \\
& +x^{2}\left[-9.44284-2.73820 \ell_{x}+6 \ell_{x}^{2}\right] \\
& +x^{3}\left[234.5798 \pm 0.0044+37.9774 \ell_{x}\right] \\
= & -0.93428-1.83496] \\
& +[-0.0137294] \\
& +[-0.0002209+0.0003415+0.0039893] \\
& +[0.0000265362 \pm 0.0000000005-0.0000229050] \\
= & -2.76923] \\
& +[-0.0137294] \\
& +[0.004110] \\
& +[0.0000036312 \pm 0.0000000005] \\
= & -2.77885 \\
= & 0.307547-0.0974404 \ell_{x}-0.458889 \ell_{x}^{2}
\end{aligned}
$$




$$
\begin{aligned}
& +x\left[-2.97459-0.822467 \ell_{x}\right] \\
& +x^{2}\left[8.84230-9.50786 \ell_{x}-5.33333 \ell_{x}^{2}\right] \\
& +x^{3}\left[-7.15473 \pm 0.00091+53.9477 \ell_{x}+4.38649 \ell_{x}^{2}\right] \\
& =[0.3075+0.5195-13.0443] \\
& +[-0.0143861+0.0212076] \\
& +[0.000207+0.001186-0.003546] \\
& +[-0.00000081-0.00003254+0.00001411] \\
& =[-12.2173]+[0.006822]+[-0.002154]+[-0.00001924] \\
& =-12.2126 \text {, } \\
& A_{2}^{(8), \mathrm{II}(\mathrm{bc} 1)}=-0.0817145 \pm 0.0000026+0.300345 \ell_{x} \\
& +x[-0.0239766] \\
& +x^{2}\left[-0.0725930 \pm 0.0000015+0.125499 \ell_{x}\right] \\
& +x^{3}[-0.323625] \\
& =[-0.081714 \pm 0.000003-1.601317] \\
& +[-0.0001160] \\
& +[-0.00000170-0.00001565] \\
& +[-0.00000003661] \\
& =[-1.683031 \pm 0.000003] \\
& +[-0.0001160] \\
& +[-0.00001735] \\
& +[-0.00000003661] \\
& =-1.683165 \pm 0.000003 \text {. }
\end{aligned}
$$

The numerically dominant class is $\mathrm{II}(\mathrm{bc} 0)$; the closed muon loop in $\mathrm{II}(\mathrm{bc} 1)$ leads again to a result which is an order of magnitude smaller. In both cases the corrections from higher orders in $x$ are very small. The result of II(a) gets a contribution of nearly $0.5 \%$ from higher orders in $x$.

The asymptotic expansion of diagram class III shows a similar behaviour: already the linear correction term is below $0.04 \%$ which is actually smaller than the numerical uncertainty from the leading contribution. The result is given by

$$
\begin{aligned}
A_{2}^{(8), \mathrm{III}=} & 1.15444 \pm 0.00446-1.80996 \ell_{x} \\
& +x[-0.849197] \\
& +x^{2}\left[-1.95556 \pm 0.00400-1.25333 \ell_{x}\right] \\
& +x^{3}\left[-20.2365-15.3527 \ell_{x}\right] \\
= & {[1.1544 \pm 0.0045+9.6500] } \\
& +[-0.004107]
\end{aligned}
$$




$$
\begin{aligned}
& +[-0.00004574 \pm 0.00000009+0.00015630] \\
& +[-0.000002289+0.000009260] \\
= & {[10.8044 \pm 0.0045] } \\
& \quad+[-0.004107] \\
& \quad+[0.00011056 \pm 0.00000009] \\
& +[0.000006970] \\
= & 10.8004 \pm 0.0045 .
\end{aligned}
$$

Diagram class IV(d) is different from the previous cases since it does not contain photon self energy insertions. As a result there is no logarithmic enhancement in the leading order term. Note that IV(d) involves also more complicated master integrals which lead to a larger relative uncertainty. The higher order corrections in $x$ are again small. We obtain

$$
\begin{aligned}
A_{2}^{(8), \mathrm{IV}(\mathrm{d})}= & -4.33491 \pm 0.06055 \\
& +x[1.61430 \pm 0.09570] \\
& +x^{2}\left[-435.46 \pm 13.85+(-135.694 \pm 0.166) \ell_{x}\right] \\
& +x^{3}\left[-328.90 \pm 24.77-614.619 \ell_{x}+39.4784 \ell_{x}\right] \\
= & -4.33491 \pm 0.06055] \\
& +[0.007807 \pm 0.000463] \\
& +[-0.010186 \pm 0.000324+(0.016922 \pm 0.000021)] \\
& +[-0.0000372 \pm 0.0000028+0.0003707+0.0001269] \\
= & -4.335 \pm 0.061] \\
& +[0.00781 \pm 0.00046] \\
& +[0.00710 \pm 0.00037] \\
& +[0.0004604 \pm 0.0000028] \\
= & -4.320 \pm 0.061 .
\end{aligned}
$$

Note that some diagram classes have been computed analytically in the limit $m_{e}=0$, with which our leading order results agree. In Ref. [56] the classes I(a0), I(a1) and the sum of $\mathrm{I}(\mathrm{bc} 0)$ and $\mathrm{II}(\mathrm{bc} 0)$ are presented. The leading order of $\mathrm{I}(\mathrm{d})$ can be found in Ref. [57].

Our final results are summarized in Table 1. As compared to the expression in the above equations, which show the one standard deviation uncertainties originating from the numerical integration, we multiply the uncertainties in Table 1 by a factor five, in order to present conservative results. In case no uncertainty is displayed the corresponding result is either known analytically or with high numerical precision.

For comparison we show in the most right column of Table 1 the results available in the literature. The most up-to-date results for the diagram classes defined in Figure 1 can be 


\begin{tabular}{|c|c|c|c|}
\hline$A_{2}^{(8)}\left(m_{\mu} / m_{e}\right)$ & this work & literature & \\
\hline $\mathrm{I}(\mathrm{a} 0)$ & 7.223076 & $\begin{array}{l}7.223077 \pm 0.000029 \\
7.223076\end{array}$ & $\begin{array}{l}30 \\
58\end{array}$ \\
\hline $\mathrm{I}(\mathrm{a} 1)$ & 0.494072 & $\begin{array}{l}0.494075 \pm 0.000006 \\
0.494072\end{array}$ & 30 \\
\hline $\mathrm{I}(\mathrm{a} 2)$ & 0.027988 & $\begin{array}{l}0.027988 \pm 0.000001 \\
0.027988\end{array}$ & 30 \\
\hline $\mathrm{I}(\mathrm{a})$ & 7.745136 & $7.74547 \pm 0.00042$ & 32 \\
\hline $\mathrm{I}(\mathrm{bc} 0)$ & $8.56876 \pm 0.00001$ & $8.56874 \pm 0.00005$ & 30 \\
\hline $\mathrm{I}(\mathrm{bc} 1)$ & $0.1411 \pm 0.0060$ & $0.141184 \pm 0.000003$ & 30 \\
\hline $\mathrm{I}(\mathrm{bc} 2)$ & $0.4956 \pm 0.0004$ & $0.49565 \pm 0.00001$ & 30 \\
\hline $\mathrm{I}(\mathrm{bc})$ & $9.2054 \pm 0.0060$ & $9.20632 \pm 0.00071$ & 32 \\
\hline $\mathrm{I}(\mathrm{d})$ & $-0.2303 \pm 0.0024$ & $\begin{array}{l}-0.22982 \pm 0.00037 \\
-0.230362 \pm 0.000005\end{array}$ & 52 \\
\hline II(a) & -2.77885 & $\begin{array}{l}-2.77888 \pm 0.00038 \\
-2.77885\end{array}$ & 52 \\
\hline$\overline{\mathrm{II}(\mathrm{bc} 0)}$ & -12.212631 & $-12.21247 \pm 0.00045$ & 30 \\
\hline $\mathrm{II}(\mathrm{bc} 1)$ & $-1.683165 \pm 0.000013$ & $-1.68319 \pm 0.00014$ & 30 \\
\hline $\mathrm{II}(\mathrm{bc})$ & $-13.895796 \pm 0.000013$ & $-13.89457 \pm 0.00088$ & 32 \\
\hline III & $10.800 \pm 0.022$ & $10.7934 \pm 0.0027$ & 32 \\
\hline $\mathrm{IV}(\mathrm{a} 0)$ & $116.76 \pm 0.02$ & $\begin{array}{l}116.759183 \pm 0.000292 \\
111.1 \pm 8.1 \\
117.4 \pm 0.5\end{array}$ & $\begin{array}{l}30 \\
60 \\
61\end{array}$ \\
\hline IV(a1) & $2.69 \pm 0.14$ & $2.697443 \pm 0.000142$ & 30 \\
\hline IV (a2) & $4.33 \pm 0.17$ & $4.328885 \pm 0.000293$ & 30 \\
\hline IV (a) & $123.78 \pm 0.22$ & $123.78551 \pm 0.00044$ & 32 \\
\hline IV(b) & $-0.38 \pm 0.08$ & $-0.4170 \pm 0.0037$ & 32 \\
\hline $\mathrm{IV}(\mathrm{c})$ & $2.94 \pm 0.30$ & $2.9072 \pm 0.0044$ & 32 \\
\hline $\mathrm{IV}(\mathrm{d})$ & $-4.32 \pm 0.30$ & $-4.43243 \pm 0.00058$ & 32 \\
\hline
\end{tabular}

Table 1: Final results for the different classes and comparison with the literature. Note that the uncertainties in the second column are multiplied by a factor five. The results for IV(a)-IV(c) have been taken over from Ref. 11.

found in Ref. [32. To obtain separate results for sub-classes we resort to Ref. [30]. We could reproduce the analytic results for some of the sub-classes which have been obtained in Ref. [58]. In addition we managed to obtain analytic results for the case II(bc0). The corresponding results are shown in Appendix $\mathrm{A}$.

We find perfect agreement of our results with the ones in the literature although in some cases the uncertainty is far below the per mille level. In our approach we find relative large uncertainties of about 10\% and 7\% for the classes IV(c) and IV(d), respectively. For 
class III the uncertainty amounts to $0.2 \%$ and for $\mathrm{I}(\mathrm{d}) 1 \%$.

Our final result for $A_{2}^{(8)}\left(m_{\mu} / m_{e}\right)$ is given by

$$
\begin{aligned}
A_{2}^{(8)} & =A_{2}^{(8), \mathrm{lbl}}+A_{2}^{(8), \mathrm{rem}} \\
& =126.34(38)+6.53(30)=132.86(48) .
\end{aligned}
$$

Our numerical uncertainty amounts to approximately $0.5 \times(\alpha / \pi)^{4} \approx 1.5 \times 10^{-11}$. It is larger than the uncertainty in Ref. [32]. Nevertheless it is sufficiently accurate as can be seen by the comparison to the difference between the experimental result and theory prediction which is given by 3

$$
a_{\mu}(\exp )-a_{\mu}(\mathrm{SM}) \approx 249(87) \times 10^{-11} .
$$

Note that the uncertainty in Eq. (8) receives approximately the same amount from experiment and theory (i.e. the hadronic contribution). Even after a projected reduction of the uncertainty by a factor four both in $a_{\mu}(\exp )$ and $a_{\mu}(\mathrm{SM})$ our numerical precision is a factor ten below the uncertainty of the difference.

\section{Simultaneous electron and tau contribution to $a_{\mu}$}

This section provides the four-loop results to $a_{\mu}$ from the Feynman diagrams which contain simultaneously a closed electron and tau loop. Such contributions arise from the diagrams classes I(a), I(b), I(c), II(b), II(c) and IV(a).

Each integral depends on all three lepton masses. As discussed in Section 2 we perform a nested asymptotic expansion to obtain a double expansion in $m_{\mu} / m_{\tau}$ and $m_{e} / m_{\mu}$. Due to the decoupling of the heavy tau lepton the whole contribution is suppressed by a factor $1 / m_{\tau}^{2}$. Furthermore, The expansion in the inverse heavy tau mass is analytic and thus produces only even powers in $m_{\tau}$. However, odd powers in $m_{\mu} / m_{\tau}$ and $m_{e} / m_{\tau}$ arise from the two-loop mass counterterm contribution [47, 54]. Thus, we obtain one more order in $1 / m_{\tau}$ for free which is taken into account in the analytic and numeric results presented below.

In the following we present analytic results for $A_{3}^{(8)}\left(m_{\mu} / m_{e}, m_{\mu} / m_{\tau}\right)$ including terms up to $\mathcal{O}\left(1 / m_{\tau}^{7}\right)$. We expand in $m_{e} / m_{\mu}$ up to the order where non-computed terms can safely be neglected. In fact, neglected terms are of order $m_{e}^{4} / m_{\mu} / m_{\tau}^{3}, m_{e}^{3} m_{\mu}^{2} / m_{\tau}^{5}$, and $m_{e}^{2} m_{\mu}^{5} / m_{\tau}^{7}$. Our result reads

$$
A_{3}^{(8)}\left(\frac{m_{\mu}}{m_{e}}, \frac{m_{\mu}}{m_{\tau}}\right)=\frac{m_{\mu}^{2}}{m_{\tau}^{2}}\left(\frac{1}{135} \ln ^{2} \frac{m_{e}^{2}}{m_{\mu}^{2}}+\frac{89}{810} \ln ^{2} \frac{m_{\mu}^{2}}{m_{\tau}^{2}}+\ln \frac{m_{\mu}^{2}}{m_{\tau}^{2}}\left(\frac{22493}{291600}-\frac{3 \zeta_{3}}{2}\right)\right.
$$

\footnotetext{
${ }^{3}$ This result is taken from Ref. 32 .
} 


$$
\begin{aligned}
& +\ln \frac{m_{e}^{2}}{m_{\mu}^{2}}\left(-\frac{23}{270} \ln \frac{m_{\mu}^{2}}{m_{\tau}^{2}}-\frac{3 \zeta_{3}}{2}+\frac{2 \pi^{2}}{45}+\frac{74597}{97200}\right) \\
& \left.+\frac{17 \zeta_{3}}{135}+\frac{2 \pi^{4}}{75}+\frac{193 \pi^{2}}{810}-\frac{984587}{486000}-\frac{8}{135} \pi^{2} \log (2)\right) \\
& +\frac{m_{e} m_{\mu}}{m_{\tau}^{2}}\left(\frac{4 \pi^{2}}{15} \ln \frac{m_{\mu}^{2}}{m_{\tau}^{2}}-\frac{821 \pi^{2}}{900}\right) \\
& +\frac{m_{e}^{2}}{m_{\tau}^{2}}\left(\ln ^{2} \frac{m_{e}^{2}}{m_{\mu}^{2}}\left(-\frac{1}{10} \ln \frac{m_{\mu}^{2}}{m_{\tau}^{2}}-\frac{\pi^{2}}{36}+\frac{17}{50}\right)\right. \\
& +\ln \frac{m_{e}^{2}}{m_{\mu}^{2}}\left(\frac{3}{5} \ln \frac{m_{\mu}^{2}}{m_{\tau}^{2}}-\frac{\zeta_{3}}{3}-\frac{4 \pi^{4}}{135}-\frac{23 \pi^{2}}{135}-\frac{673}{450}\right) \\
& +\left(-\frac{43}{45}-\frac{\pi^{2}}{15}\right) \ln \frac{m_{\mu}^{2}}{m_{\tau}^{2}}-\frac{16 \zeta_{5}}{9}-\frac{8 \pi^{2} \zeta_{3}}{15}+\frac{47 \zeta_{3}}{45} \\
& \left.-\frac{251 \pi^{4}}{4050}+\frac{56 \pi^{2}}{225}+\frac{45671}{8100}-\frac{4}{15} \pi^{2} \log (2)\right) \\
& +\frac{m_{e}^{3}}{m_{\mu} m_{\tau}^{2}}\left(\frac{28 \pi^{3}}{135}-\frac{64 \pi^{2}}{135} \ln \frac{m_{e}^{2}}{m_{\mu}^{2}}-\frac{4 \pi^{2}}{135} \ln \frac{m_{\mu}^{2}}{m_{\tau}^{2}}-\frac{5689 \pi^{2}}{24300}\right) \\
& +\frac{\pi^{2}}{90} \frac{m_{e} m_{\mu}^{2}}{m_{\tau}^{3}} \\
& +\frac{m_{\mu}^{4}}{m_{\tau}^{4}}\left(\ln ^{2} \frac{m_{e}^{2}}{m_{\mu}^{2}}\left(\frac{1}{420} \ln \frac{m_{\mu}^{2}}{m_{\tau}^{2}}+\frac{3}{19600}\right)+\frac{1181}{40824} \ln ^{3} \frac{m_{\mu}^{2}}{m_{\tau}^{2}}\right. \\
& +\ln \frac{m_{e}^{2}}{m_{\mu}^{2}}\left(\frac{4553}{90720} \ln ^{2} \frac{m_{\mu}^{2}}{m_{\tau}^{2}}-\frac{1074457}{4762800} \ln \frac{m_{\mu}^{2}}{m_{\tau}^{2}}-\frac{1811 \zeta_{3}}{2304}\right. \\
& \left.+\frac{1811 \pi^{2}}{68040}+\frac{2304926093}{2667168000}\right)-\frac{3034811}{38102400} \ln ^{2} \frac{m_{\mu}^{2}}{m_{\tau}^{2}} \\
& +\ln \frac{m_{\mu}^{2}}{m_{\tau}^{2}}\left(-\frac{61849 \zeta_{3}}{80640}+\frac{3520386679}{4000752000}-\frac{2011 \pi^{2}}{204120}\right) \\
& -\frac{9564133 \zeta_{3}}{76204800}+\frac{50467 \pi^{4}}{2903040}+\frac{9308371 \pi^{2}}{85730400} \\
& -\frac{9932011422817}{5040947520000}-\frac{7}{1152} \log ^{4}(2) \\
& \left.+\frac{7 \pi^{2}}{1152} \log ^{2}(2)-\frac{44}{945} \pi^{2} \log (2)-\frac{7 a_{4}}{48}\right) \\
& +\frac{m_{e} m_{\mu}^{3}}{m_{\tau}^{4}}\left(\frac{19 \pi^{2}}{720} \ln \frac{m_{\mu}^{2}}{m_{\tau}^{2}}-\frac{2161 \pi^{2}}{43200}\right) \\
& +\frac{m_{e}^{2} m_{\mu}^{2}}{m_{\tau}^{4}}\left(\ln ^{2} \frac{m_{e}^{2}}{m_{\mu}^{2}}\left(\frac{61}{3528}-\frac{1}{84} \ln \frac{m_{\mu}^{2}}{m_{\tau}^{2}}\right)-\frac{3}{280} \ln ^{3} \frac{m_{\mu}^{2}}{m_{\tau}^{2}}\right.
\end{aligned}
$$




$$
\begin{aligned}
& +\ln \frac{m_{e}^{2}}{m_{\mu}^{2}}\left(-\frac{9}{280} \ln ^{2} \frac{m_{\mu}^{2}}{m_{\tau}^{2}}+\frac{49181}{176400} \ln \frac{m_{\mu}^{2}}{m_{\tau}^{2}}+\frac{\pi^{2}}{168}\right. \\
& \left.-\frac{5938843}{12348000}\right)+\frac{130813}{1058400} \ln ^{2} \frac{m_{\mu}^{2}}{m_{\tau}^{2}} \\
& +\ln \frac{m_{\mu}^{2}}{m_{\tau}^{2}}\left(\frac{\zeta_{3}}{70}-\frac{1050211}{1543500}+\frac{17 \pi^{2}}{1260}\right) \\
& \left.+\frac{6751 \zeta_{3}}{14700}+\frac{\pi^{4}}{1050}-\frac{163823 \pi^{2}}{1587600}-\frac{271714897}{23337720000}\right) \\
& +\frac{m_{e} m_{\mu}^{4}}{m_{\tau}^{5}}\left(\frac{\pi^{2}}{140} \ln \frac{m_{\mu}^{2}}{m_{\tau}^{2}}+\frac{79 \pi^{2}}{19600}\right) \\
& +\frac{m_{\mu}^{6}}{m_{\tau}^{6}}\left(\ln ^{2} \frac{m_{e}^{2}}{m_{\mu}^{2}}\left(\frac{2}{945} \ln \frac{m_{\mu}^{2}}{m_{\tau}^{2}}-\frac{131}{297675}\right)\right. \\
& +\ln \frac{m_{e}^{2}}{m_{\mu}^{2}}\left(\frac{20929}{604800} \ln ^{2} \frac{m_{\mu}^{2}}{m_{\tau}^{2}}-\frac{229507}{1984500} \ln \frac{m_{\mu}^{2}}{m_{\tau}^{2}}\right. \\
& \left.-\frac{3077 \zeta_{3}}{5760}+\frac{83 \pi^{2}}{4800}+\frac{84725571607}{160030080000}\right) \\
& +\frac{15787}{777600} \ln ^{3} \frac{m_{\mu}^{2}}{m_{\tau}^{2}}-\frac{104754659}{2286144000} \ln ^{2} \frac{m_{\mu}^{2}}{m_{\tau}^{2}} \\
& +\ln \frac{m_{\mu}^{2}}{m_{\tau}^{2}}\left(-\frac{62761 \zeta_{3}}{120960}+\frac{33670638521}{51438240000}-\frac{3973 \pi^{2}}{544320}\right) \\
& -\frac{34589999 \zeta_{3}}{152409600}+\frac{818557 \pi^{4}}{65318400}+\frac{43896581 \pi^{2}}{685843200} \\
& -\frac{34820138253959}{28355329800000}-\frac{17}{2880} \log ^{4}(2)+\frac{17 \pi^{2}}{2880} \log ^{2}(2) \\
& \left.-\frac{512 \pi^{2}}{14175} \log (2)-\frac{17 a_{4}}{120}\right) \\
& +\frac{m_{e} m_{\mu}^{5}}{m_{\tau}^{6}}\left(\frac{11 \pi^{2}}{5670} \ln \frac{m_{\mu}^{2}}{m_{\tau}^{2}}-\frac{39157 \pi^{2}}{3572100}\right) \\
& +\frac{m_{e} m_{\mu}^{6}}{m_{\tau}^{7}}\left(\frac{\pi^{2}}{105} \ln \frac{m_{\mu}^{2}}{m_{\tau}^{2}}+\frac{79 \pi^{2}}{66150}\right)+\ldots
\end{aligned}
$$

For the numerical evaluation of our result we use 62

$$
\begin{aligned}
& m_{\mu} / m_{e}=206.7682843(52), \\
& m_{\mu} / m_{\tau}=5.94649(54) \cdot 10^{-2},
\end{aligned}
$$

and obtain

$$
A_{3, \mu}^{(8)}\left(m_{\mu} / m_{e}, m_{\mu} / m_{\tau}\right) \approx 0.06321803-0.00049494-0.00000111
$$




\begin{tabular}{crc} 
& \multicolumn{2}{c}{$A_{3}^{(8)}\left(m_{\mu} / m_{e}, m_{\mu} / m_{\tau}\right)$} \\
\hline group & this work & Ref. [32] \\
\hline I(a) & $0.00320905(1)$ & $0.003209(0)$ \\
I(b) + I(c) & $0.00442289(2)$ & $0.004422(0)$ \\
II(b) + II(c) & $-0.02865753(1)$ & $-0.028650(2)$ \\
IV(a) & $0.08374757(9)$ & $0.083739(36)$
\end{tabular}

Table 2: Comparison of our results to Ref. [32] for the individual diagram classes. Note that the error in $m_{\mu} / m_{\tau}$ is not included. In most cases it induces an uncertainty in fifth significant digit in the displayed numbers, in the cass of IV(a) even in the fourth. In the second row an updated result for I(c) has been used, see footnote 4.

$$
=0.0627220(1)(100)
$$

where the three numbers in the first line are obtained as follows: The first number includes terms up to $\mathcal{O}\left(m_{\tau}^{-3}\right)$ with numerator factors $m_{e}^{0}$ or $m_{e}^{1}$. All quadratic corrections in $m_{e}$ are part of the second term which also contains all $\mathcal{O}\left(m_{\tau}^{-4}\right)$ and $\mathcal{O}\left(m_{\tau}^{-5}\right)$ corrections. The last term in the first line of (11) comprises the remaining contributions of order $\mathcal{O}\left(m_{\tau}^{-6}\right)$ and $\mathcal{O}\left(m_{\tau}^{-7}\right)$ and the cubic electron mass terms. In the second line the final result is presented. The first uncertainty is set to $10 \%$ of the last expansion term in the first line which is our estimate for the missing higher order terms. The second error originates from the experimental uncertainties for $m_{\mu} / m_{\tau}$ and $m_{\mu} / m_{e}$ as given above. The result is in good agreement with $A_{3}^{(8)}\left(m_{\mu} / m_{e}, m_{\mu} / m_{\tau}\right)=0.06272(4)$ [32].

Although the absolute contribution from $A_{3}^{(8)}$ is quite small it is nevertheless instructive to compare our results to the complete numerical method of Ref. 32. This is done in Table 2 for the individual diagram classes contributing to $A_{3}^{(8)}\left(m_{\mu} / m_{e}, m_{\mu} / m_{\tau}\right)$ 出 Good agreement within the uncertainties are found for the diagram classes I(a) and IV(a). For II(b)+II(c) one observes a discrepancy of about three standard deviations. Also the results for $\mathrm{I}(\mathrm{b})+\mathrm{I}(\mathrm{c})$ do not agree within the assigned uncertainty. Note, however, that in 32 an older value for $m_{\mu} / m_{\tau}$ has been used which is about $0.01 \%$ smaller and thus can explain most of the discrepancy.

\section{Conclusions}

We presented results for the complete four-loop electron contribution to the anomalous magnetic moment of the muon. This includes light-by-light-type contributions (which have already been presented in Ref. [1]), the remaining contributions where the external

\footnotetext{
${ }^{4}$ We thank Makiko Nio for providing us with an updated result for the contribution I(c).
} 
photon couples to the (external) muon line (see Section 4), and the contribution which involves both electron and tau loops (see Section 5 ).

Our calculation serves as an important cross check to the calculation performed in Ref. 32] which is based on an entire numerical method. In our approach we express $a_{\mu}$ in terms of an analytic linear combination of four-loop master integrals. Thus, our result can be improved systematically by evaluating more and more master integrals either analytically or with higher numerical precision.

We have demonstrated that our current (numerical) result for $a_{\mu}$ is sufficiently precise: even after reducing the current experimental and theory uncertainty by a factor four the uncertainty induced by our four-loop expression is a factor ten smaller.

As a by-product we have computed three-loop corrections to the mass and wave function on-shell renormalization constants of the muon taking into account effects from a finite electron mass.

Note that the four-loop contribution involving heavy tau loops have been computed in Ref. 33. Thus, only the four-loop universal part remains to be cross checked.

The four-loop results of Refs. [1,33] and this paper can be summarized as

$$
a_{\mu}^{(8)}=\left.a_{\mu}^{(8)}\right|_{\text {univ. }}+132.86(48)+0.0424941(53)+0.062722(10)
$$

where the second third and fourth term on the right-hand side corresponds to $A_{2}^{(8)}\left(m_{\mu} / m_{e}\right)$, $A_{2}^{(8)}\left(m_{\mu} / m_{\tau}\right)$ and $A_{3}^{(8)}\left(m_{\mu} / m_{e}, m_{\mu} / m_{\tau}\right)$, respectively.

\section{Acknowledgments}

We would like to thank M. Nio for useful communications concerning the results from Ref. [32]. We thank the High Performance Computing Center Stuttgart (HLRS) and the Supercomputing Center of Lomonosov Moscow State University [63] for providing computing time used for the numerical computations with FIESTA. P.M was supported in part by the EU Network HIGGSTOOLS PITN-GA-2012-316704. The work of V.S. was supported by the Alexander von Humboldt Foundation (Humboldt Forschungspreis).

\section{A Analytic results for selected contributions}

In this appendix we present results for the diagram classes $\mathrm{I}(\mathrm{a})$, II(a) and II(bc). The expansion coefficients are either known analytically or to high numerical precision. They 
are given by

$$
\begin{aligned}
& A_{2}^{(8), \mathrm{I}(\mathrm{a} 0)}=-\frac{8609}{5832}-\frac{25 \pi^{2}}{162}-\frac{2 \zeta_{3}}{9}+\left(-\frac{317}{162}-\frac{2 \pi^{2}}{27}\right) \ell_{x}-\frac{25}{27} \ell_{x}^{2}-\frac{4}{27} \ell_{x}^{3} \\
& +6.405155074501456 x \\
& +x^{2}\left(\frac{967}{315}+\frac{26 \pi^{2}}{27}+\frac{136 \zeta_{3}}{35}+\left(\frac{304}{27}+\frac{8 \pi^{2}}{9}\right) \ell_{x}+\frac{52}{9} \ell_{x}^{2}+\frac{16}{9} \ell_{x}^{3}\right) \\
& -52.00224911971472 x^{3} \\
& A_{2}^{(8), \mathrm{I}(\mathrm{a} 1)}=\frac{7627}{1944}+\frac{13 \pi^{2}}{27}-\frac{4 \pi^{4}}{45}+\ell_{x}\left(\frac{61}{81}-\frac{2 \pi^{2}}{27}\right)+\ell_{x}^{2}\left(\frac{119}{27}-\frac{4 \pi^{2}}{9}\right) \\
& +x^{2}\left(\frac{227}{18}-\frac{4 \pi^{2}}{3}+\ell_{x}\left(\frac{230}{27}-\frac{8 \pi^{2}}{9}\right)\right) \\
& A_{2}^{(8), \mathrm{I}(\mathrm{a} 2)}=0.0007061505929186751+\ell_{x}\left(\frac{943}{162}+\frac{8 \pi^{2}}{135}-\frac{16 \zeta_{3}}{3}\right) \\
& +0.004933870387556993 x^{2} \\
& A_{2}^{(8), \mathrm{II}(\mathrm{a})}=-0.9342776853294055+\ell_{x}\left(\frac{31}{16}-\frac{5 \pi^{2}}{12}+\frac{\pi^{2} \log (2)}{2}-\frac{3 \zeta_{3}}{4}\right) \\
& +x\left(\frac{79 \pi^{2}}{54}-\frac{13 \pi^{3}}{36}-\frac{8 \pi^{2} \log (2)}{9}\right) \\
& +x^{2}(-9.442838330353652 \\
& \left.+\ell_{x}\left(-\frac{47}{2}+\frac{37 \pi^{2}}{4}-14 \pi^{2} \log (2)+21 \zeta_{3}\right)+6 \ell_{x}^{2}\right) \\
& A_{2}^{(8), \mathrm{II}(\mathrm{bc} 0)}=\frac{2299 \pi^{2}}{648}-\frac{17233}{1728}+\frac{40 a_{4}}{3}+\frac{16 a_{5}}{3}-\frac{37 \zeta_{5}}{6}-\frac{\pi^{2} \zeta_{3}}{8}+\frac{431 \zeta_{3}}{36} \\
& -\frac{403 \pi^{4}}{3240}-\frac{2 \log ^{5}(2)}{45}+\frac{5 \log ^{4}(2)}{9}-\frac{4}{27} \pi^{2} \log ^{3}(2) \\
& -\frac{31}{540} \pi^{4} \log (2)-\frac{235}{54} \pi^{2} \log (2)+\frac{10}{9} \pi^{2} \log ^{2}(2) \\
& +\left(\frac{16 a_{4}}{3}+7 \zeta_{3}-\frac{11 \pi^{4}}{108}+\frac{79 \pi^{2}}{27}-\frac{115}{12}\right. \\
& \left.+\frac{2 \log ^{4}(2)}{9}+\frac{4}{9} \pi^{2} \log ^{2}(2)-\frac{10}{3} \pi^{2} \log (2)\right) \ell_{x} \\
& +\left(\zeta_{3}-\frac{31}{12}+\frac{5 \pi^{2}}{9}-\frac{2}{3} \pi^{2} \log (2)\right) \ell_{x}^{2}+x\left(-\frac{217 \pi^{2}}{720}-\frac{\pi^{2}}{12} \ell_{x}\right) \\
& +x^{2}\left(\frac{425}{36}-\frac{112 a_{4}}{3}-\frac{455 \zeta_{3}}{12}+\frac{335 \pi^{4}}{432}-\frac{409 \pi^{2}}{36}\right. \\
& -\frac{14 \log ^{4}(2)}{9}-\frac{28}{9} \pi^{2} \log ^{2}(2)+\frac{299}{18} \pi^{2} \log (2)
\end{aligned}
$$




$$
\left.+\left(\frac{13}{3}-14 \zeta_{3}-\frac{37 \pi^{2}}{6}+\frac{28}{3} \pi^{2} \log (2)\right) \ell_{x}-\frac{16}{3} \ell_{x}^{2}\right)
$$

Note that analytic results for I(a0), I(a1), I(a2) and II(a) can be found in Ref. [58].

\section{References}

[1] A. Kurz, T. Liu, P. Marquard, A. V. Smirnov, V. A. Smirnov and M. Steinhauser, Phys. Rev. D 92 (2015) 7, 073019 doi:10.1103/PhysRevD.92.073019 arXiv:1508.00901 [hep-ph]].

[2] G. W. Bennett et al. [Muon g-2 Collaboration], Phys. Rev. D 73 (2006) 072003 [hep-ex/0602035].

[3] B. L. Roberts, Chin. Phys. C 34 (2010) 741 arXiv:1001.2898 [hep-ex]].

[4] M. Davier, A. Hoecker, B. Malaescu and Z. Zhang, Eur. Phys. J. C 71 (2011) 1515 [Erratum-ibid. C 72 (2012) 1874] arXiv:1010.4180 [hep-ph]].

[5] K. Hagiwara, R. Liao, A. D. Martin, D. Nomura and T. Teubner, J. Phys. G 38 (2011) 085003 [arXiv:1105.3149 [hep-ph]].

[6] F. Jegerlehner and R. Szafron, Eur. Phys. J. C 71 (2011) 1632 arXiv:1101.2872 [hep$\mathrm{ph}]]$.

[7] M. Benayoun, P. David, L. DelBuono and F. Jegerlehner, Eur. Phys. J. C 73 (2013) 2453 arXiv:1210.7184 [hep-ph]].

[8] B. Krause, Phys. Lett. B 390 (1997) 392 [hep-ph/9607259].

[9] D. Greynat and E. de Rafael, JHEP 1207 (2012) 020 [arXiv:1204.3029 [hep-ph]].

[10] K. Hagiwara, A. D. Martin, D. Nomura and T. Teubner, Phys. Rev. D 69 (2004) 093003 [hep-ph/0312250].

[11] A. Kurz, T. Liu, P. Marquard and M. Steinhauser, Phys. Lett. B 734 (2014) 144 arXiv:1403.6400 [hep-ph]].

[12] A. Nyffeler, Phys. Rev. D 79 (2009) 073012 [arXiv:0901.1172 [hep-ph]].

[13] K. Melnikov and A. Vainshtein, Phys. Rev. D 70 (2004) 113006 [hep-ph/0312226].

[14] J. Bijnens and J. Prades, Mod. Phys. Lett. A 22 (2007) 767 hep-ph/0702170 [HEP$\mathrm{PH}]$.

[15] G. Colangelo, M. Hoferichter, A. Nyffeler, M. Passera and P. Stoffer, Phys. Lett. B 735 (2014) 90 [arXiv:1403.7512 [hep-ph]]. 
[16] A. Czarnecki, B. Krause and W. J. Marciano, Phys. Rev. Lett. 76 (1996) 3267 [hep-ph/9512369|.

[17] M. Knecht, S. Peris, M. Perrottet and E. De Rafael, JHEP 0211 (2002) 003 hep-ph/0205102.

[18] A. Czarnecki, W. J. Marciano and A. Vainshtein, Phys. Rev. D 67 (2003) 073006 [Phys. Rev. D 73 (2006) 119901] [hep-ph/0212229].

[19] C. Gnendiger, D. Stöckinger and H. Stöckinger-Kim, Phys. Rev. D 88 (2013) 053005 [arXiv:1306.5546 [hep-ph]].

[20] J. S. Schwinger, Phys. Rev. 73 (1948) 416.

[21] A. Petermann, Helv. Phys. Acta 30 (1957) 407.

[22] C. M. Sommerfield, Phys. Rev. 107 (1957) 328.

[23] H.H. Elend, Phys. Lett. 20 (1966) 682; 21 (1966) 720(E).

[24] M. A. Samuel and G. -w. Li, Phys. Rev. D 44 (1991) 3935 [Erratum-ibid. D 48 (1993) 1879].

[25] S. Laporta and E. Remiddi, Phys. Lett. B 301 (1993) 440.

[26] S. Laporta, Nuovo Cim. A 106 (1993) 675.

[27] S. Laporta and E. Remiddi, Phys. Lett. B 379 (1996) 283 [hep-ph/9602417].

[28] A. Czarnecki and M. Skrzypek, Phys. Lett. B 449 (1999) 354 hep-ph/9812394.

[29] M. Passera, Phys. Rev. D 75 (2007) 013002 hep-ph/0606174.

[30] T. Kinoshita and M. Nio, Phys. Rev. D 70 (2004) 113001 hep-ph/0402206].

[31] T. Aoyama, M. Hayakawa, T. Kinoshita and M. Nio, Phys. Rev. D 77 (2008) 053012 arXiv:0712.2607 [hep-ph]].

[32] T. Aoyama, M. Hayakawa, T. Kinoshita and M. Nio, Phys. Rev. Lett. 109 (2012) 111808 [arXiv:1205.5370 [hep-ph]].

[33] A. Kurz, T. Liu, P. Marquard and M. Steinhauser, Nucl. Phys. B 879 (2014) 1 arXiv:1311.2471 [hep-ph]].

[34] M. Beneke and V. A. Smirnov, Nucl. Phys. B 522 (1998) 321 doi:10.1016/S05503213(98)00138-2 hep-ph/9711391].

[35] V. A. Smirnov, "Applied asymptotic expansions in momenta and masses," Springer Tracts Mod. Phys. 177 (2002) 1. 
[36] A. Pak and A. Smirnov, Eur. Phys. J. C 71 (2011) 1626 [arXiv:1011.4863 [hep-ph]].

[37] B. Jantzen, A. V. Smirnov and V. A. Smirnov, Eur. Phys. J. C 72 (2012) 2139 arXiv:1206.0546 [hep-ph]].

[38] V. A. Smirnov, Springer Tracts Mod. Phys. 250 (2012) 1. doi:10.1007/978-3-64234886-0

[39] J. A. M. Vermaseren, arXiv:math-ph/0010025.

[40] J. Kuipers, T. Ueda, J. A. M. Vermaseren and J. Vollinga, Comput. Phys. Commun. 184 (2013) 1453 [arXiv:1203.6543 [cs.SC]].

[41] M. Tentyukov and J. A. M. Vermaseren, Comput. Phys. Commun. 181 (2010) 1419 [hep-ph/0702279].

[42] M. Steinhauser, T. Ueda and J. A. M. Vermaseren, Nucl. Part. Phys. Proc. 261-262 45 [arXiv:1501.07119 [hep-ph]].

[43] A. V. Smirnov, Comput. Phys. Commun. 189 (2014) 182 arXiv:1408.2372 [hep-ph]].

[44] P. Marquard, D. Seidel, unpublished.

[45] P. Marquard, A. V. Smirnov, V. A. Smirnov and M. Steinhauser, Phys. Rev. Lett. 114 (2015) 14, 142002 arXiv:1502.01030 [hep-ph]].

[46] A. V. Smirnov, Comput. Phys. Commun. 185 (2014) 2090 arXiv:1312.3186 [hep-ph]].

[47] N. Gray, D. J. Broadhurst, W. Grafe and K. Schilcher, Z. Phys. C 48 (1990) 673.

[48] D. J. Broadhurst, N. Gray and K. Schilcher, Z. Phys. C 52 (1991) 111.

[49] K. Melnikov and T. v. Ritbergen, Phys. Lett. B 482 (2000) 99 [hep-ph/9912391.

[50] P. Marquard, L. Mihaila, J. H. Piclum and M. Steinhauser, Nucl. Phys. B 773 (2007) 1 hep-ph/0702185.

[51] K. Melnikov and T. van Ritbergen, Nucl. Phys. B 591 (2000) 515 hep-ph/0005131.

[52] K. G. Chetyrkin and M. Steinhauser, Phys. Rev. Lett. 83 (1999) 4001 hep-ph/9907509.

[53] K. G. Chetyrkin and M. Steinhauser, Nucl. Phys. B 573 (2000) 617 [hep-ph/9911434].

[54] S. Bekavac, A. Grozin, D. Seidel and M. Steinhauser, JHEP 0710 (2007) 006 arXiv:0708.1729 [hep-ph]].

[55] S. Bekavac, A. G. Grozin, D. Seidel and V. A. Smirnov, Nucl. Phys. B 819 (2009) 183 doi:10.1016/j.nuclphysb.2009.04.015 [arXiv:0903.4760 [hep-ph]]. 
[56] R. Lee, P. Marquard, A. V. Smirnov, V. A. Smirnov and M. Steinhauser, JHEP 1303 (2013) 162 [arXiv:1301.6481 [hep-ph]].

[57] D. J. Broadhurst, A. L. Kataev and O. V. Tarasov, Phys. Lett. B 298 (1993) 445 hep-ph/9210255.

[58] S. Laporta, Phys. Lett. B 312 (1993) 495 hep-ph/9306324.

[59] P. A. Baikov and D. J. Broadhurst, hep-ph/9504398.

[60] J. Calmet and A. Peterman, Phys. Lett. B 56 (1975) 383.

[61] C. Chlouber and M. A. Samuel, Phys. Rev. D 16 (1977) 3596.

[62] J. Beringer et al. [Particle Data Group Collaboration], Phys. Rev. D 86 (2012) 010001.

[63] V. Sadovnichy, A. Tikhonravov, V. Voevodin, and V. Opanasenko, "Lomonosov': Supercomputing at Moscow State University." In Contemporary High Performance Computing: From Petascale toward Exascale (Chapman \& Hall/CRC Computational Science), pp.283-307, Boca Raton, USA, CRC Press, 2013. 